\title{
NV-5138 as a fast-acting antidepressant via direct activation of mTORC1 signaling
}

\author{
Yuto Hasegawa, Xiaolei Zhu, and Atsushi Kamiya \\ Department of Psychiatry and Behavioral Sciences, Johns Hopkins University School of Medicine, Baltimore, Maryland, USA.
}

\begin{abstract}
Growing evidence implicates altered mTORC1 signaling cascades in the pathophysiology of depression, suggesting that direct modulation of mTORC1 signaling may offer novel therapeutic potential. In this issue of the $J C I$, Kato and colleagues reported that administration of NV-5138, a recently developed synthetic leucine analog, has a rapid and sustained antidepressant action in rat models via activation of mTORC1 signaling. The investigators also found that the antidepressant effect of NV-5138 is mediated by upregulation of brain-derived neurotrophic factor (BDNF) signaling and that NV-5138 treatment produces rapid synaptic responses in the medial prefrontal cortex. These findings highlight the direct activation of mTORC1 signaling as a potential pharmacological intervention for the treatment of depression.
\end{abstract}

by two critical downstream substrates of mTOR complex 1 (mTORC1), the p70 ribosomal S6 kinases 1 and 2 (p70S6K1/2), and the eukaryotic initiation factor $4 \mathrm{E}$-binding proteins (4E-BPs) $(6,7)$. It has been reported that the acute antidepressant action of ketamine is accompanied by elevated synaptic protein expression and upregulation of mTOR-S6K1 and 4E-BP signaling cascades in rats $(8,9)$. Interestingly, fast-acting antidepressant effects of ketamine are also dependent on the rapid increase of brain-derived neurotrophic factor (BDNF) production via inactivation of eukaryotic elongation factor 2 (eEF2) kinase, a substrate of S6K1 (10). Thus, although more studies are needed to understand how altered mTOR signaling is involved in the pathophysiology of depression, mTORC1 signaling is a unique molecular target that can be used to explore drugs with rapid antidepressant action, such as ketamine.

\section{Rapid antidepressant action} via direct activation of mTORC1 signaling

mTORC1 signaling is regulated by the interaction between sestrins and the GATOR2 complex (11). Leucine, a critical amino acid in mTORC1-mediated protein synthesis, can bind sestrins, resulting in release from GATOR2, leading to mTORC1 activation (12). NV-5138 is a novel synthetic analog of leucine that has sufficient oral bioavailability and brain penetration to selectively bind sestrins for the activation of mTORC1 signaling in the brain (13). In this issue of the $J C I$, Kato et al. report that oral administration of NV-5138 has a rapid and prolonged antidepressant action in rat models (14). They systematically demonstrate a rapid and long-lasting antidepressant effect of a single dose of NV-5138 in multiple depression-associated behavioral paradigms, including the forced swim test, the novelty suppressed feed test, and the female urine sniffing test in wild type rats. They also show that NV-5138 rapidly ameliorated chronic unpredictable stress-induced 


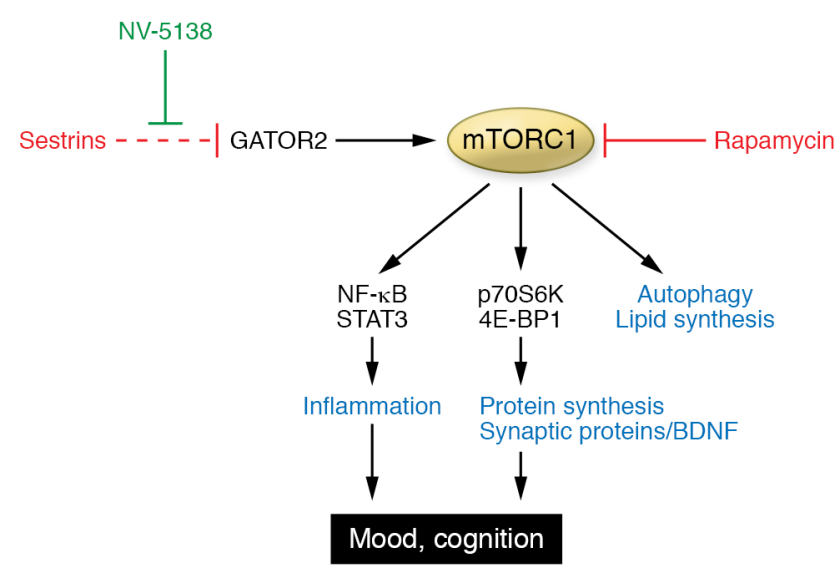

Figure 1. Antidepressant action of NV-5138 via direct activation of mTORC1 signaling. Rapamycinsensitive mTORC1 signaling regulates the production of synaptic proteins and BDNF via p70S6K and 4E-BP1 signaling pathways. NV-5138, a synthetic leucine analog, binds sestrins and releases sestrins from GATOR2, resulting in mTORC1 activation and producing fast antidepressant effects. Note that mTORC1 signaling also regulates inflammatory machinery via NF- $\kappa B$ and STAT3 signaling pathways, suggesting that aberrant inflammatory mechanisms underlying depressive symptoms may also be targetable via modulation of mTORC1 signaling.

anhedonia-like behaviors in a sucrose preference test. Additionally, they confirmed that NV-5138, similarly to ketamine, rapidly upregulated phosphorylation of mTOR and increased phospho-p70s6K1 and $4 \mathrm{E}-\mathrm{BP} 1$, which are downstream targets of mTORC1 in the prefrontal cortex. Importantly, intrainfusion of rapamycin, a selective mTORC1 inhibitor, blocked the antidepressant effect of NV-5138. These results suggest that activation of mTORC1 signaling is a promising approach for the treatment of depressive symptoms and that NV-5138 is a promising lead compound for the development of new drugs with rapid antidepressant action.

\section{Rapid synaptic changes and upregulation of BDNF signaling by mTORC 1 activation}

Kato et al. also examined the effect of NV-5138 on synaptic function by conducting biochemical, immunohistochemical, and electrophysiological experiments. Chronic unpredictable stress exposure resulted in the reduction of GluR1 and PSD95 in the synaptosome fraction of the medial prefrontal cortex, which was rapidly reversed by a single dose of NV-5138 treatment. Morphological analysis of dendritic spine in the pyramidal neurons in layer $\mathrm{V}$ of the medial prefrontal cortex showed an increase in spine density in the rats subjected to acute administration of a single dose of NV-5138 compared with control groups. To examine the influence of NV-5138 treatment on synaptic function, 5-HT and hypocretin-induced excitatory postsynaptic current (EPSC) response in the layer $\mathrm{V}$ pyramidal neurons was measured by whole-cell electrophysiology in rat prefrontal cortex brain slices 24 hours after a single dose of NV-5138 or vehicle treatment. Consistent with the ketamine effect on synaptic function $(8,9,15)$, NV-5138 treatment led to a significant increase in the frequency and amplitude of 5-HT and hypocretin-induced EPSC in neurons compared with that in controls. Expression of synaptic proteins, such as GluR1, Synapsin 1, and SV2A, was increased by NV-5138 treatment, which was similar to the effect of ketamine. These results suggest that NV-5138 and ketamine may, at least in part, share the common mechanisms underlying pharmacological effects on synaptic function, leading to a rapid antidepressant effect. This idea is supported by the results showing that intrainfusion of an antibody against BDNF in the medial prefrontal cortex 30 minutes before NV-5138 administration blocked acute antidepressant behavioral responses induced by a single dose of NV-5138. Consistent with the above, the BDNF knockin mice with a genetic variant of BDNF (Met66Met), a polymorphism reported to inhibit BDNF processing (16), suppressed the antidepressant behavioral effect of NV-5138.

\section{Conclusions and future directions}

In this study, Kato and colleagues reported, for the first time, that direct pharmacological activation of mTORC1 signaling led to a rapid and sustained antidepressant effect. Notably, the impact of NV-5138 on synaptic function and BDNF signaling is similar to that of ketamine, suggesting that NV-5138 and ketamine, at least in part, share the common mTORC1 signaling-mediated mechanisms underlying their antidepressant actions. Paradoxically, however, a recent clinical trial revealed that pretreatment with the mTORC1 inhibitor rapamycin prolonged the antidepressant effect of ketamine in MDD patients (17), underscoring the need for further investigation for understanding precise mechanisms of the modulation of mTORC1 signaling by ketamine. With the authors having shown that ketamine induces a rapid antidepressant effect in treatment-resistant patients (4), the antidepressant effect of NV-5138 reported in this study may translate to efficacy for patients, such as those with treatment-resistance MDD, who respond to ketamine. Nonetheless, considering that mTOR is a ubiquitously expressed serine/ threonine kinase and plays essential roles for multiple cellular functions, future studies should be carefully conducted to determine whether NV-5138 induces any adverse effects, including the promotion of carcinogenesis. There are emerging links between CNS inflammation and glutamate signaling underlying depression (18). Furthermore, a recent study reported that pharmacological inhibition of microglial glutamate production ameliorates chronic stress-induced depressive-like behaviors (19). Considering that mTOR signaling also regulates the immune and inflammatory systems via modulation of NF- $\mathrm{kB}$ and STAT3 signaling pathways (ref. 20 and Figure 1), targeting mTORC1 signaling in brain immune cells also warrants future investigation.

\section{Acknowledgments}

We thank Aisa Moreno-Megui for critical reading of this Commentary. Work in the Kamiya laboratory is supported by NIH grants, including R01DA041208 (to AK) and P5OMH094268 (to AK).

Address correspondence to: Atsushi Kamiya, Department of Psychiatry and Behav- 
ioral Sciences, Johns Hopkins University School of Medicine, 600 North Wolfe Street, Meyer 3-146, Baltimore, Maryland 21287, USA. Phone: 410.502 .0060 ; Email: akamiya1@jhmi.edu.

1. Hasin DS, et al. Epidemiology of adult DSM-5 major depressive disorder and its specifiers in the United States. JAMA Psychiatry. 2018;75(4):336-346.

2. Lieblich SM, Castle DJ, Pantelis C, Hopwood M, Young AH, Everall IP. High heterogeneity and low reliability in the diagnosis of major depression will impair the development of new drugs. BJPsych Open. 2015;1(2):e5-e7.

3. Mathew SJ, Manji HK, Charney DS. Novel drugs and therapeutic targets for severe mood disorders. Neuropsychopharmacology. 2008;33(9):2080-2092.

4. Zarate CA Jr, et al. A randomized trial of an $\mathrm{N}$-methyl-D-aspartate antagonist in treatmentresistant major depression. Arch Gen Psychiatry. 2006;63(8):856-864.

5. Berman RM, et al. Antidepressant effects of ketamine in depressed patients. Biol Psychiatry. 2000;47(4):351-354.

6. Bockaert J, Marin P. mTOR in brain physiology and pathologies. Physiol Rev. 2015;95(4):1157-1187. 7. Lipton JO, Sahin M. The neurology of mTOR. Neuron. 2014;84(2):275-291.

8. Li N, et al. mTOR-dependent synapse formation underlies the rapid antidepressant effects of NMDA antagonists. Science. 2010;329(5994):959-964.

9. Li N, et al. Glutamate N-methyl-D-aspartate receptor antagonists rapidly reverse behavioral and synaptic deficits caused by chronic stress exposure. Biol Psychiatry. 2011;69(8):754-761.

10. Autry AE, et al. NMDA receptor blockade at rest triggers rapid behavioural antidepressant responses. Nature. 2011;475(7354):91-95.

11. Chantranupong $\mathrm{L}$, et al. The Sestrins interact with GATOR2 to negatively regulate the aminoacid-sensing pathway upstream of mTORC1. Cell Rep. 2014;9(1):1-8.

12. Wolfson RL, et al. Sestrin2 is a leucine sensor for the mTORC1 pathway. Science. 2016;351(6268):43-48.

13. Sengupta S, et al. Discovery of NV-5138, the first selective Brain mTORC1 activator. Sci Rep. 2019;9(1):4107.

14. Kato T, et al. Sestrin modulator NV-5138 produces rapid antidepressant effects via direct mTORC1 activation. JClin Invest.
2019;129(6):2542-2554.

15. Fukumoto K, et al. Activity-dependent brainderived neurotrophic factor signaling is required for the antidepressant actions of $(2 R, 6 R)$ hydroxynorketamine. Proc Natl Acad Sci US A. 2019;116(1):297-302.

16. Chen ZY, et al. Genetic variant BDNF (Val66Met) polymorphism alters anxiety-related behavior. Science. 2006;314(5796):140-143.

17. Abdallah CG, et al. Rapamycin, an immunosuppressant mTORC1 inhibitor, triples theantidepressant response rate of ketamine at 2 weeks following treatment. bioRxiv Website. https://www.biorxiv.org/content/10.1101/500959v1. Published December 19, 2018. Accessed April 24, 2019.

18. Haroon E, Miller AH, Sanacora G. Inflammation, glutamate, and glia: a trio of trouble in mood disorders. Neuropsychopharmacology. 2017;42(1):193-215.

19. Zhu X, et al. JHU-083 selectively blocks glutaminase activity in brain $\mathrm{CD} 11 \mathrm{~b}(+)$ cells and prevents depression-associated behaviors induced by chronic social defeat stress. Neuropsychopharmacology. 2019;44(4):683-694.

20. Dazert E, Hall MN. mTOR signaling in disease. Curr Opin Cell Biol. 2011;23(6):744-755. 